\title{
Implementation of IoT based Smart Laboratory
}

\author{
M. Poongothai \\ Department of ECE \\ Coimbatore Institute of \\ Technology \\ Coimbatore-641014
}

\author{
A. L. Karupaiya \\ Department of ECE \\ Coimbatore Institute of \\ Technology \\ Coimbatore-641014
}

\author{
R. Priyadharshini \\ Department of ECE \\ Coimbatore Institute of \\ Technology \\ Coimbatore-641014
}

\begin{abstract}
Internet of Things is a promising technology that has wasted no time spreading across the world and connecting the huge number of individuals with the devices around them. The proposed work aims to develop a smart laboratory system in CIT campus based on IoT and mobile application technologies that operates in an intelligent manner. This provides a platform that allows devices to be connected, sensed and controlled remotely across a network infrastructure. The IoT lab is implemented by embedding the electrical appliances such as lights, air-conditioners, fans and projectors in the laboratory with sensors and network connectivity. These software enabled physical objects collect and exchange the real time data. Devices in the laboratory are connected to IoT smart hardware kit and communicate through an MQTT protocol which is a messaging protocol for machine-to-machine communication. IoT smart hardware kits are designed using ESP8266, Arduino UNO, relays and current transformers. All ESP8266s act as MQTT clients and an online server acts as an MQTT broker. The proposed work mainly intents to provide an easy accessibility of the electrical appliances through an Android application and a user interactive dashboard developed in Node-RED. Along with the status and energy consumption of individual devices, temperature \& humidity status of the laboratory can also be monitored using sensors and viewed in dashboard and mobile application. From the results of implementation, it is observed that the appliances in our lab are remotely monitored and controlled, thereby reducing their energy consumption considerably.
\end{abstract}

\section{Keywords}

Environmental monitoring, IoT kit, Mobile application, MQTT, Node-RED, User- interactive dashboard

\section{INTRODUCTION}

The Internet of things is a communication paradigm that refers to the idea of connecting the objects of everyday life to the internet. These objects are assembled with microcontrollers, transceivers to enable communication, and configured with protocol stacks that will realize the interaction of the objects with one another to reach to common goals without human intervention This paradigm gained its strength from the fact that it is interacting with a wide variety of devices such as: robots, drones, heating and air-conditioning systems, security alarms, household appliances, power generation systems, office equipment, and so on, which generate a massive amount of data to provide new services to people and both public and private sectors[2]. A smart laboratory system is based on IoT and mobile application technologies and aims to provide a practice platform for CIT students learning IoT concepts and mobile application technology. It provides a real smart environment for CIT to help improve campus management in the Internet era and also provides security, energy efficiency and comfort to the end user. As campus becomes bigger, new management problem appears. Managing the huge number of electrical systems and devices in a campus has become a real problem. There also arises a problem in power management. It is difficult to monitor all sub-systems such as lighting, projecting and air conditioning system. But if these systems are left indiscriminately, energy will be wasted. The proposed work aims to reduce the manual effort by automating laboratory resources, thereby achieving a futuristic model of laboratory using IoT. This can be achieved by replacing some traditional devices by the smart IoT hardware kit so that subsystem devices will connect to each other for better access[3]. The user can check the status of the light and control the electrical appliances remotely. These devices provide benefits to the users with limited mobility that may have a difficult time getting to or even reaching their control switch.

\section{RELATED WORKS}

An automation system uses the portable devices as a user interface. They can communicate with an automation network through an Internet gateway, by means of low power communication protocols like Wi-Fi [5]. The emerging technologies of Internet of Things (IoT) can be utilized to better manage energy consumption. In the following, few related research works in this field are briefly discussed.

Chandramohan et al [1] proposed the intelligent operation for lamps and fans. Here the system is connected with temperature control and lamp control. Light dependent resistor (LDR) and Temperature sensor (LM35) are the main components for this automatic control of lamps and fans. In this work, the LDR is responsible for lamp control and LM35 is responsible for controlling the operation of fan. The proposed home energy control system design intelligent services for users and provides comfort. The system proposed in this work [1] is implemented with smart phone.

Pavithra.D et al [9] proposed an efficient implementation for IoT (Internet of Things) used for monitoring and controlling the home appliances via World Wide Web. This work aims at controlling home appliances via Smartphone using Wi-Fi as communication protocol and raspberry pi as server system. The user here moves directly with the system through a webbased interface over the web, whereas home appliances like lights, fan and door lock are remotely controlled through easy website. The server is interfaced with relay hardware circuits that control the appliances running at home. The communication with server allows the user to select the appropriate device. The communication with server permits the user to pick out the acceptable device. The server communicates with the corresponding relays. If the web affiliation is down or the server isn't up, the embedded system board still will manage and operate the appliances domestically. The proposed concept is extremely clichéd and lacks sound design principles. 
Akbar Satria et al [12] reveal that most of the home automation systems use Bluetooth in their mobile device for implementation. This has various demerits in every aspect. Primarily the Bluetooth has a very small range and the proposed system could only work in small homes, excluding homes with larger area. Bluetooth does not support remote operations, (i.e.) the appliances cannot be controlled by Bluetooth from a remote geographical area. Many appliances within the same range may pick up the commands given to particular device resulting in erroneous behaviour.

Kodali R.K et al [10] presented an overview of a light weight Message Queuing Telemetry Transport (MQTT) protocol. In the prototype, author attempted to implement MQTT on ESP8266, a WiFi based development board. Sensors and actuators are connected to ESP8266 and a Mosquitto based MQTT broker is established for remote monitoring and control.

Vamsikrishna Patchava et al [11] have proposed a system for Smart Home Automation technique with Raspberry Pi using IoT and it is done by integrating cameras and motion sensors into a web application. This system has been designed using a Raspberry Pi module with Computer Vision techniques. Using this, home appliances connected through a monitor based internet can be controlled. Raspberry Pi operates and controls motion sensors and video cameras for sensing and surveillance. For instance, it captures intruder's identity and detects its presence using simple Computer Vision Technique (CVT). Whenever motion is detected, the cameras start recording and Raspberry Pi device alerts the owner through an SMS and alarm call.

\section{PROPOSED MODEL}

The proposed work aims at designing a smart laboratory that facilitates remote monitoring and control of the lab devices using mobile application and Node-RED dashboard. In this system, each lab device is interfaced with a data acquisition module that is an IoT object with a unique IP address resulting in a mesh wireless network of devices. The data acquisition System on Chip (SoC) module collects energy consumption data from each device of smart lab and transmits the data to a centralized server for further processing and analysis[7]. All appliances in the laboratory use MQTT (Message Queuing Telemetry Transport) protocol for communication.

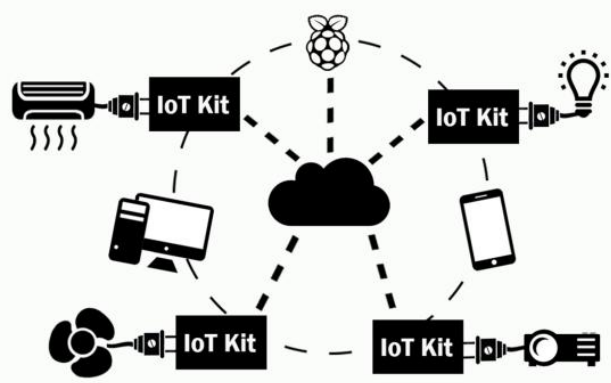

\section{Figure 1: Block diagram of the proposed system}

MQTT is a machine-machine connectivity protocol specially used in Internet of Things application. It is designed as an extremely lightweight publish/subscribe messaging transport. All systems in the laboratory use this protocol for communication. The systems are connected to ESP8266s which act as MQTT clients. An online server acts as an MQTT broker [6].

Node-RED is a programming tool for wiring hardware devices. It provides a browser-based editor that makes it easy to wire wide range of nodes in the palette that can be deployed to its runtime in a single-click. Node-RED application aids in creating the interactive user interface dashboards. RASPBERRY PI 3 [4] runs the Node-RED application in it. An android application is developed using Android Studio that provides accessibility to control the devices. The control signals are sent from the broker through the dashboard or mobile application. Clients publish the status of the devices to the broker and can be viewed in the dashboard. All the devices can be controlled universally and the status of the devices can be visualized.

\subsection{Hardware Setup}

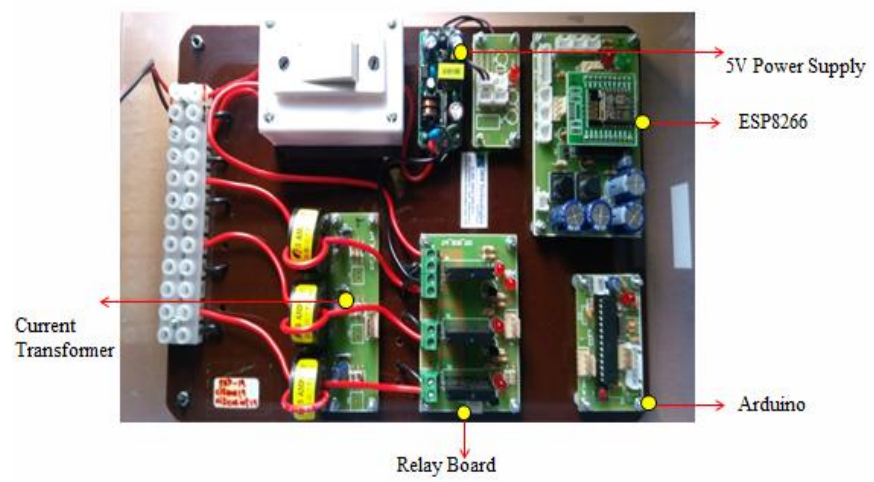

Figure 2: Hardware setup of IoT kit

The main components used are

- $\quad$ ESP8266- It is a low-cost Wi-Fi microchip with full TCP/IP stack and microcontroller capability. Here, it acts as an MQTT client.

- Arduino UNO- It is a microcontroller board based on the ATmega328P. Here, it sends the status of the devices to client.

- Current Transformer- It is a transformer that measures the AC current of the circuit. It senses the current flowing through it and the values are in analog.

- Relay- It acts as a protective switch. It uses an electromagnetic switch operated by a small electric current that can turn on or off a much larger current [8].

\section{RESULTS AND DISCUSSION}

Using this system, lights and fans are controlled and their usage can be monitored by the user using his mobile phone. This system ensures optimal utilization of energy by continuous monitoring of the lab environment by integrating the lab devices through an IoT platform. The devices are monitored continuously and their energy consumption and usage are calculated and displayed in the Node-RED dashboard. 


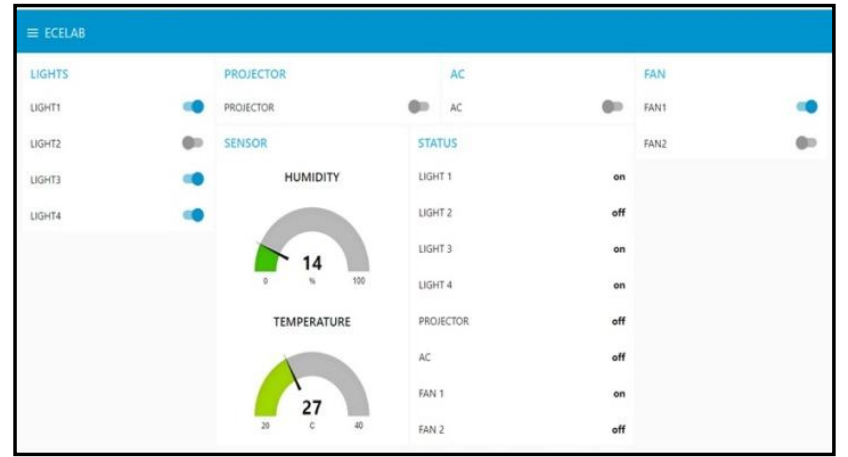

Figure 3: Dashboard for ECE Lab

Figure 3 shows the dashboard that displays the temperature and humidity conditions inside the laboratory that are measured by sensors connected to NODE-MCU which acts as a client. The Node-RED dashboard acts as an output window that shows the actual status of all the connected devices and provides access to the device which is shown in Figure 4. The mobile application has been developed that allows controlling the entire appliances all at a same time and also the individual device.

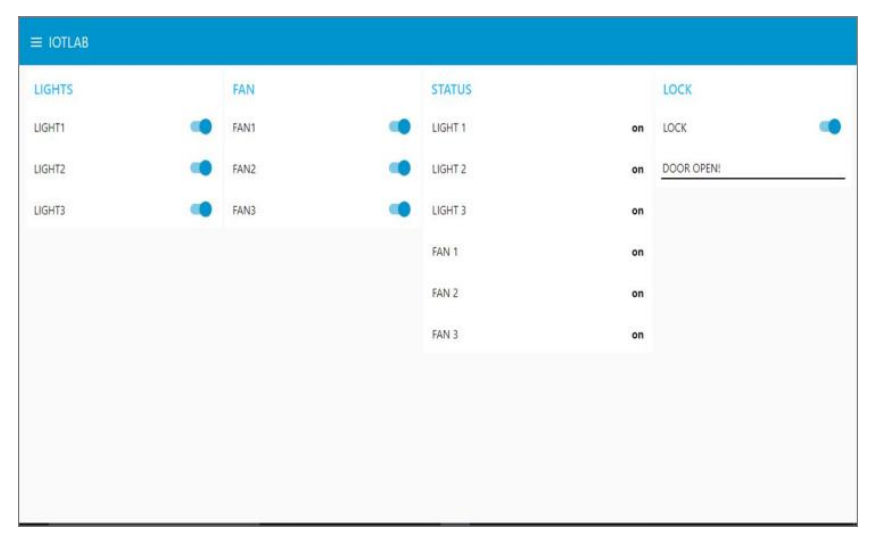

Figure 4: Dashboard for IoT Lab

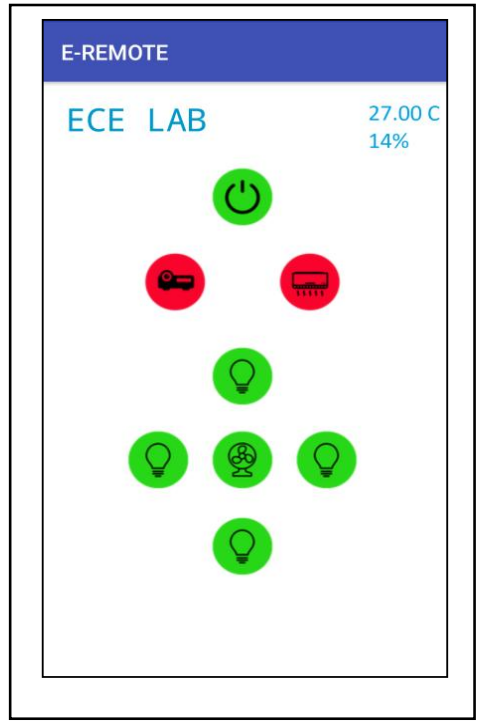

Figure 5a

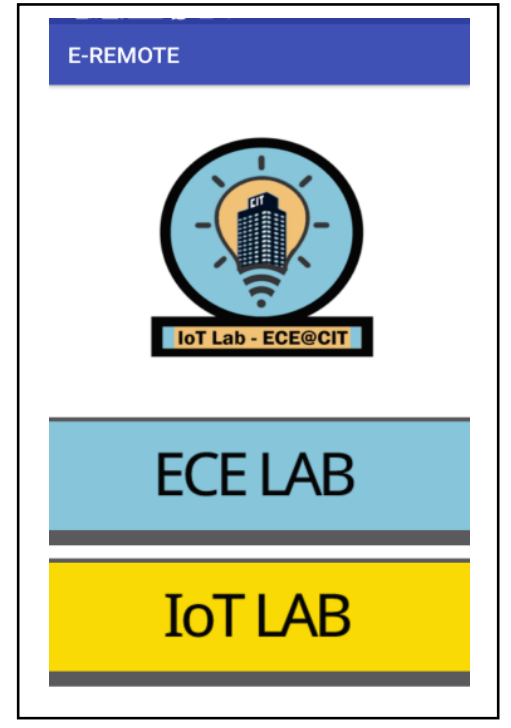

Figure 5b

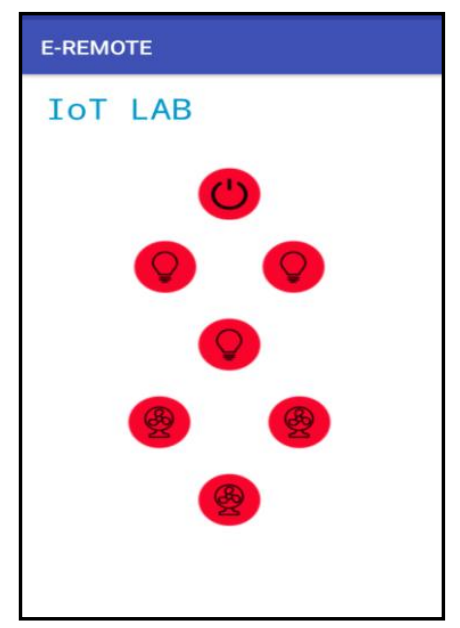

Figure 5c

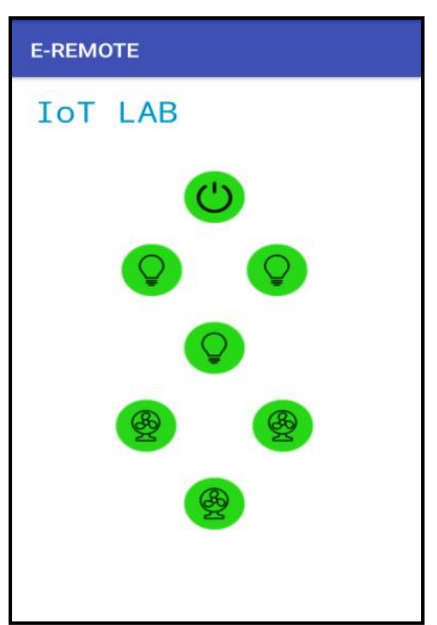

Figure 5d

Figure 5: E-REMOTE app pages

E-Remote is a locally developed interactive application for android users. This application has been developed in ANDROID STUDIO using JAVA programming language. It is a digital remote to monitor and control the appliances of IoT Lab and ECE Lab which is shown in Fig. 5. The proposed 
system has been implemented in IOT lab@CIT of ECE department and thus the results are obtained.

\section{CONCLUSION}

In this work, the proposed system facilitates the users to control the appliances such as lights, fans, projector and airconditioner just by giving commands either through a smart phone or Node-RED Dashboard. Thus all devices can be controlled universally, and the status of the devices can be visualized. This feature will help the user to analyze the status of these devices anytime and anywhere. It reduces human intervention in monitoring the devices. This system can be further extended for automatic ON and OFF of appliances depending upon the number of users in the Lab using Omron thermal sensors. This system can be further implemented in hospitals for physically challenged people for controlling the appliances.

\section{ACKNOWLEDGMENTS}

This work is financially supported by the Coimbatore Institute of Technology. The authors would like to acknowledge Dr.V.Selladurai, Principal, CIT and Dr. A,Rajeswari , Professor and Head, Department of ECE, CIT for their guidance and support throughout the period of this work.

\section{REFERENCES}

[1] ChandraMohan,R.Nagarajan,K.Satheeshkumar,N.AjithK umar,P.A.Gopinath,S.RanjithKumar . 2017 "Intelligent Smart Home Automation and Security System using Arduino and Wi-Fi" , International Journal of Engineering and Computer Science, vol 6,issue 3,pp.20694-20698.

[2] Chunsheng Zhu, Victor C.M. Leung, Lei Shu . 2015 "Green Internet of Things for Smart World", IEEE, Volume: 3,Issue : 2, pp.2151-2162.

[3] HafsaTahir, Ayesha Kanwer and M. Junaid . 2016, "Internet of Things (IoT): An Overview of Applications and Security Issues Regarding Implementation", International Journal Of Multidisciplinary Sciences and Engineering, , Vol. 7, No. 1.

[4] Jagdish A. Patel, AringaleShubhangi, Shweta Joshi , AartiPawar, Namrata Bari . 2016,"Raspberry PI Based Smart Home", Internation Journal of Engineering Science and computing,Vol.6, No.3..
[5] Mary Cherian, Hitesh Kumar P . 2014, "Implementation of a Secure and Smart Lab with Wireless Sensor Network", International Journal of Science and Research, Vol.3, No. 6.

[6] SiddharthLodha, SapnaTiwari, RajashriPatil, KedarnathShinde, Prof. E. Jayanthi . 2015 "Mobile Based Home Automation System using Iot and Prediction Algorithm", International Education \& Research Journal, Volume : 3,Issue : 5,pp.68-69.

[7] Supriya Sonar, MayuriMujmule, Tejashwini Mangalgire, Prof. Thawali.B.R . 2016 "Home Automation using IoT", International Journal of Engineering and Computer Science,vol6,issue 4,pp. 20923-20926

[8] Vinay sagar K N,Kusuma S M . 2015, "Home Automation Using Internet of Things", International Research Journal of Engineering and Technology, Vol. 2, No.3.

Pavithra, D. and Balakrishnan, R . 2015, "IoT based monitoring and control system for home automation",IEEE Global Conference Communication Technologies (GCCT) on 2015, pp 169-173.

[9] Kodali, R.K. and Soratkal, S. 2016, "MQTT based home automation system using ESP8266", IEEE Region 10 conference In Humanitarian Technology (R10-HTC), pp. $1-5$.

[10] Kelly, S. D. T., Suryadevara, N. K., \&Mukhopadhyay, S. C. 2013, "Towards the implementation of IoT for environmental condition monitoring in homes",IEEE Sensors Journal, vol.13, issue 10, pp.3846-3853.

[11] Javale, D., Mohsin, M., Nandanwar, S. and Shingate M. 2013, "Home automation and security system using Android ADK", International journal of electronics communication and computer technology (IJECCT), vol.3, issue 2, pp.382-385.

[12] Satria, A., Priadi, M.L., Wulandhari, L.A. and Budiharto, W. 2015, "The Framework of Home Remote Automation System Based on Smartphone",International journal of smart home, vol.9, issue 1, pp.53-60.

[13] https://nodered.org/

[14] https://nodemcu.readthedocs.io/en/master/en/modules/gp io/ 